\title{
ON THE DERIVATIVES AT THE ORIGIN OF ENTIRE HARMONIC FUNCTIONS
}

\author{
by D. H. ARMITAGE
}

(Received 11 January, 1978)

1. Introduction. If $f$ is an entire function in the complex plane such that

$$
\max _{|z|=r}|f(z)|=O\left(e^{\alpha r}\right) \quad(r \rightarrow \infty),
$$

where $0 \leq \alpha<1$, and all the derivatives of $f$ at 0 are integers, then it is easy to show that $f$ is a polynomial (see e.g. Straus [10]). The best possible result of this type was proved by Pólya [9]. The main aim of this paper is to prove two analogous results for harmonic functions defined in the whole of the Euclidean space $\mathbf{R}^{n}$, where $n \geq 2$ (i.e. entire harmonic functions).

Before stating the main results, we give some notations. A point of $\mathbf{R}^{n}$ is denoted by $X=\left(x_{1}, \ldots, x_{n}\right)$. Throughout the paper $a$ denotes an $n$-tuple $\left(a_{1}, \ldots, a_{n}\right)$ of non-negative integers, and we put

$$
|a|=a_{1}+\ldots+a_{n}, \quad a !=a_{1} ! \ldots a_{n} !
$$

and

$$
D^{a}=\left(\frac{\partial}{\partial x_{1}}\right)^{a_{1}} \ldots\left(\frac{\partial}{\partial x_{n}}\right)^{a_{n}} .
$$

We shall use $m$ consistently to denote a non-negative integer. If $f$ is an infinitely differentiable function in an open subset of $\mathbf{R}^{n}$, the norm of the gradient of order $m$ of $f$ is defined by

$$
\left|\nabla_{m} f\right|=\left\{m ! \sum_{|a|=m}\left(D^{a} f\right)^{2}(a !)^{-1}\right\}^{1 / 2}
$$

Thus $\left|\nabla_{0} f\right|=|f|$ and $\left|\nabla_{1} f\right|$ is the usual norm of the gradient (of order 1 ) of $f$. Also, it is easy to show that

$$
\left|\nabla_{m} f\right|=\left\{\sum_{b_{1}=1}^{n} \ldots \sum_{b_{m}=1}^{n}\left(\partial^{m} f / \partial x_{b_{1}} \ldots \partial x_{b_{m}}\right)^{2}\right\}^{1 / 2}
$$

(see Calderón and Zygmund [3]), whence it follows that if $h$ is harmonic in $\mathbf{R}^{\mathbf{n}}$, then

$$
\left|\nabla_{m} h\right|^{2}=2^{-m} \Delta^{m}\left(h^{2}\right),
$$

Glasgow Math. J. 20 (1979) 147-154. 
where $\Delta^{m}$ is the $m$ th iterated Laplacian operator in $\mathbf{R}^{n}$. In particular, we note that by (1) our definition of $\left|\nabla_{m} f\right|$ agrees with that given by Kuran [6]. We denote the origin of $\mathbf{R}^{n}$ by $O$. If $r$ is a positive number, the open ball and the sphere of centre $O$ and radius $r$ in $\mathbf{R}^{n}$ are denoted by $B(r)$ and $S(r)$ respectively. If a function $g$ is defined and continuous at least on $S(r)$, then the mean of $g$ over $S(r)$ is given by the equation

$$
\mathcal{M}(g, r)=\left(s_{n} r^{n-1}\right)^{-1} \int_{S(r)} g d \sigma,
$$

where $\sigma$ is the surface area measure on $S(r)$ and $s_{n}$ is the surface area of $S(1)$.

THEOREM 1. Let $h$ be harmonic in $\mathbf{R}^{n}$ and suppose that

$$
\mathcal{M}(|h|, r)=O\left(e^{\alpha r}\right) \quad(r \rightarrow \infty)
$$

where $0 \leq \alpha<1$. If $D^{a} h(O)$ is an integer for each $n$-tuple a, then $h$ is a polynomial. The result is false with $\alpha=1$.

THEOREM 2. Let $h$ be harmonic in $\mathbf{R}^{n}$ and suppose that (3) holds for some $\alpha$ such that $0 \leq \alpha<1 / \sqrt{2}$. If $\left|\nabla_{m} h(O)\right|$ is an integer for all $m$, then $h$ is a polynomial. The result is false with $\alpha=1 / \sqrt{2}$.

It will become obvious that, in proving Theorem 1 , we need only suppose that $D^{a} h(O)$ is an integer when $a$ is sufficiently large. Similarly, in Theorem 2 we need only suppose that $\left|\nabla_{m} h(O)\right|$ is an integer for all sufficiently large $m$. In fact, in Theorem 1 , we require only that there is a positive integer $p$ such that $D^{a} h(O)$ is an integer whenever $a_{2}+\ldots+a_{n} \geq p$ and $a_{1}=0$ or 1 , for the identity $\Delta^{1} D^{a} h \equiv 0$, which holds for each $a$, will then imply that $D^{a} h(O)=0$ for any a such that $|a|>p$.

Theorems 1 and 2 will follow easily from the following lemmas respectively.

LEMMA 1. If $h$ is harmonic in $\mathbf{R}^{n}$ and (3) holds for some non-negative number $\alpha$, then

$$
D^{a} h(O)=O\left(|a|^{n-3 / 2} \alpha^{|a|}\right) \quad(|a| \rightarrow \infty)
$$

LEMMA 2. If $h$ is harmonic in $\mathbf{R}^{n}$ and (3) holds for some non-negative number $\alpha$, then

$$
\left|\nabla_{m} h(O)\right|=O\left(m^{3 n / 4-1}(\alpha \sqrt{2})^{m}\right) \quad(m \rightarrow \infty) .
$$

The special case of Lemma 1 in which $a_{2}=\ldots=a_{m}=0$ (so that $D^{a} h$ is an $x_{1}$ derivative) was proved in [1].

2. Preliminary results. In this section we reduce the proofs of Lemmas 1 and 2 to problems about harmonic polynomials.

The Poisson kernel of $B(r)$ is the function $K_{r}$, defined in $B(r) \times S(r)$ by the equation

$$
K_{r}(X, Y)=\left(s_{n} r\right)^{-1}\left(r^{2}-|X|^{2}\right)|X-Y|^{-n}
$$


where

$$
|X|=\left(x_{1}^{2}+\ldots+x_{n}^{2}\right)^{1 / 2}
$$

It is well known that if $h$ is harmonic in an open set containing the closure $\bar{B}(r)$ of $B(r)$, then

$$
h(X)=\int_{S(r)} K_{r}(X, Y) h(Y) d \sigma(Y) \quad(X \in B(r))
$$

(see e.g. Helms [5, p. 16]). Since $K_{r}$ and all its partial derivatives with respect to $x_{1}, \ldots, x_{n}$ are continuous in $B(r) \times S(r)$, we have

$$
D^{a} h(O)=\int_{S(r)} D^{a} K_{r}(O, Y) h(Y) d \sigma(Y)
$$

for any $a$. The main problem thus becomes that of estimating $D^{a} K_{r}(O, Y)$, and this will be solved by expressing $K_{r}(\cdot, Y)$ as a series of harmonic polynomials and studying the terms of this series.

The vector space of all homogeneous harmonic polynomials of degree $m$ in $\mathbf{R}^{n}$ is denoted by $\mathscr{H}_{m}$. (Note that $0 \in \mathscr{H}_{m}$ ). Brelot and Choquet [2] introduced the norm \|\| on $\mathscr{H}_{m}$, defined by the equation

$$
\|H\|=\left\{\left(s_{n}\right)^{-1} \int_{S(1)} H^{2} d \sigma\right\}^{1 / 2} .
$$

We shall need the following results.

Theorem A. If $Y \in \mathbf{R}^{n} \backslash\{O\}$, then there exists a unique element $I_{m, Y}$ (a Brelot-Choquet axial polynomial) of $\mathscr{H}_{m}$ such that $I_{m, Y}$ is invariant under rotation about the line OY (i.e. for each orthonormal transformation $T$ of $\mathbf{R}^{n}$ for which $T(Y)=Y$, we have $I_{m, Y} \circ T=I_{m, Y}$ ) and

$$
I_{m . Y}(Y)=|Y|^{m}
$$

The polynomial $I_{m, Y}$ is given in $\mathbf{R}^{n} \backslash\{O\}$ by the equation

$$
I_{m . Y}(X)=|X|^{m} P_{m}(t)
$$

where

$$
t=\left(x_{1} y_{1}+\ldots+x_{n} y_{n}\right)(|X||Y|)^{-1}
$$

and $P_{m}$ is the $n$-dimensional Legendre polynomial of degree $\boldsymbol{m}$. Further,

$$
\left\|I_{m, Y}\right\|^{2}=\left(\operatorname{dim} \mathscr{H}_{m}\right)^{-1}=(N(m, n))^{-1} \text {, say. }
$$


Most of this theorem can be found in [2]. The relation (6) is well known (see e.g. Müller [8]).

Theorem B. The Poisson kernel $K_{\mathrm{r}}$ is given in $B(r) \times S(r)$ by the equation

$$
K_{r}(X, Y)=\left(s_{n} r^{n-1}\right)^{-1} \sum_{k=0}^{\infty} N(k, n) r^{-k} I_{k, Y}(X)
$$

When $X=O$ this equation is trivial. When $X \neq O$, we deduce it from (4), (6) and the equation

$$
\sum_{k=0}^{\infty} N(k, n) u^{k} P_{k}(t)=\left(1-u^{2}\right)\left(1+u^{2}-2 u t\right)^{-n / 2} \quad(0 \leq u<1,-1 \leq t \leq 1)
$$

(see e.g. [8, p. 30]) by taking $u=|X| / r$ and $t$ to be given by (7).

LEMMA 3. If $h$ is harmonic in an open set containing $\bar{B}(r)$, then

$$
D^{a} h(O)=\left(s_{n} r^{n-1}\right)^{-1} N(|a|, n) r^{-|a|} \int_{S(r)} D^{a} I_{|a|, Y} h(Y) d \sigma(Y) .
$$

From (4) and (8), we easily obtain

$$
D^{a} h(O)=\left(s_{n} r^{n-1}\right)^{-1} \int_{S(r)} D^{a}\left\{\sum_{k=0}^{\infty} N(k, n) r^{-k} I_{k, Y}(O)\right\} h(Y) d \sigma(Y) .
$$

Clearly

$$
D^{a} I_{k, Y}(O)=0 \quad(k \neq|a|), \quad D^{a} I_{|a|, Y} \equiv D^{a} I_{|a|, Y}(O) .
$$

Hence, to prove the lemma, it is enough to show that the operator $D^{a}$ can be taken inside the summation in (10). Now, for each fixed $Y$ on $S(r)$, the function $K_{r}(\cdot, Y)$ is harmonic in $B(r)$ and therefore real-analytic in $B(r)$. Hence $K_{r}(\cdot, Y)$ is equal to its multiple Taylor series about $O$ in some neighbourhood of $O$. Bracketing together terms of equal degree in this Taylor series, we obtain a series of homogeneous polynomials, convergent to $K_{r}(\cdot, Y)$ in some neighbourhood of $O$. Since such a series is unique, it is equal term-by-term to the right-hand side of (9). Since the Taylor series can be differentiated term-by-term arbitrarily often, so also can the series in (10).

3. Harmonic polynomials. In view of Lemma 1 , our interest now turns to the estimation of the partial derivatives of $I_{m, Y}$ at $O$.

LEMma 4. If $H \in \mathscr{H}_{m}$ and $|a|=m$, then

$$
\left|D^{a} H\right| \leq m !(N(m, n))^{1 / 2}\|H\|,
$$


and in particular

$$
\left|D^{a} I_{m, Y}\right| \leq m !
$$

for each $Y$ in $\mathbf{R}^{n} \backslash\{O\}$.

When $m=0$ the lemma is trivial. For positive values of $m$, we appeal to the inequality

$$
N(m-1, n)\left\|\frac{\partial H}{\partial x_{i}}\right\|^{2} \leq m^{2} N(m, n)\|H\|^{2} \quad(i=1, \ldots, n) .
$$

This inequality is implicit in the work of Calderón and Zygmund [3, Chapter 1]. (To deduce (11) from their work one needs an explicit formula for $N(m, n)$, for which see e.g. [8].) Kuran [7, p. 17] gives (11) explicitly together with the cases of equality. Observing that each of the operators $\partial / \partial x_{i}(i=1, \ldots, n)$ maps $\mathscr{H}_{m}$ into $\mathscr{H}_{m-1}$ and using (11) repeatedly, we find that

$$
N(0, n)\left\|D^{a} H\right\|^{2} \leq(m !)^{2} N(m, n)\|H\|^{2} .
$$

Since $N(0, n)=1$ and $D^{a} H \equiv\left\|D^{a} H\right\|$, the main result of the lemma now follows. The special case where $H=I_{m, Y}$ comes from the main result and (8).

Lemma 5. If $H \in \mathscr{H}_{m}$, then

In particular,

$$
\left|\nabla_{m} H\right|=\{m ! n(n+2) \ldots(n+2 m-2)\}^{1 / 2}\|H\| .
$$

$$
\left|\nabla_{m} I_{m, Y}\right|=\left\{m ! n(n+2) \ldots(n+2 m-2)(N(m, n))^{-1}\right\}^{1 / 2}
$$

for each $Y$ in $\mathbf{R}^{n} \backslash\{O\}$.

When $m=0$ the lemma is trivial. For positive values of $m$, we use a result of Kuran [7; Lemma 2] which states that if $Q$ is a homogeneous polynomial of degree $2 m$ in $\mathbf{R}^{n}$, then

$$
\Delta^{m} Q=2^{m} m !\{n(n+2) \ldots(n+2 m-2)\}\left(s_{n}\right)^{-1} \int_{s(1)} Q d \sigma .
$$

Applying this equation with $Q=H^{2}$ and using (2), we obtain the main result of the lemma, from which by using (8) we obtain the particular result for $H=I_{m, Y}$.

4. Proof of Lemmas 1 and 2. To prove Lemma 1, we have, by Lemmas 3 and 4, for each positive number $r$

$$
\begin{aligned}
\left|D^{a} h(O)\right| & \leq\left(s_{n}\right)^{-1} r^{-|a|-n+1} N(|a|, n) \int_{S(r)}\left|D^{a} I_{|a|, Y} h(Y)\right| d \sigma(Y) \\
& \leq r^{-|a|} N(|a|, n)|a| ! M(|h|, r) \\
& \leq A r^{-|a|} N(|a|, n)|a| ! e^{\alpha r}
\end{aligned}
$$


where $A$ is the constant implied by the $O$-notation in (3). Now, there is a constant $C$, depending only on $n$, such that

$$
N(m, n) \leq C m^{n-2} \quad(m \geq 1) .
$$

Hence

$$
\left|D^{a} h(O)\right| \leq A C r^{-|a|}|a|^{n-2}|a| ! e^{\alpha r} \quad(|a| \geq 1, r>0) .
$$

In particular, taking $r=|a| / \alpha$, we obtain

$$
\left|D^{a} h(O)\right| \leq A C|a|^{n-2}|a| !(\alpha e)^{|a|}|a|^{-|a|} \quad(|a| \geq 1),
$$

and the theorem now follows by an application of Stirling's formula.

To prove Lemma 2, we have, by Lemma 1 and the Cauchy-Schwarz inequality, for each positive number $r$

$$
\begin{aligned}
\left|\nabla_{m} h(O)\right|= & \left(s_{n} r^{n-1}\right)^{-1} N(m, n) m ! r^{-m}\left\{\sum_{|a|=m}(a !)^{-1}\left(\int_{S(r)} D^{a} I_{m, Y} h(Y) d \sigma(Y)\right)^{2}\right\}^{1 / 2} \\
& \leq\left(s_{n} r^{n-1}\right)^{-1} N(m, n) m ! r^{-m}\left\{\sum_{|a|=m}(a !)^{-1} \int_{S(r)}\left(D^{a} I_{m, Y}\right)^{2}|h(Y)| d \sigma(Y)\right. \\
& \left.\times \int_{S(r)}|h(Y)| d \sigma(Y)\right\}^{1 / 2} \\
= & \left(s_{n} r^{n-1}\right)^{-1} N(m, n) r^{-m}\left\{\int_{S(r)}\left|\nabla_{m} I_{m, Y}\right|^{2}|h(Y)| d \sigma(Y)\right. \\
& \left.\times \int_{S(r)}|h(Y)| d \sigma(Y)\right\}^{1 / 2} .
\end{aligned}
$$

By Lemma 5, we now have

$$
\begin{aligned}
\left|\nabla_{m} h(O)\right| & \leq\{m ! n(n+2) \ldots(n+2 m-2) N(m, n)\}^{1 / 2} r^{-m} M(|h|, r) \\
& \leq A\left\{C m ! n(n+2) \ldots(n+2 m-2) m^{n-2}\right\}^{1 / 2} r^{-m} e^{\alpha r},
\end{aligned}
$$

where $A$ and $C$ are as before. Hence, taking $r=m / \alpha$, we obtain

$$
\begin{aligned}
\left|\nabla_{m} h(O)\right| & \leq A\left\{C m ! n(n+2) \ldots(n+2 m-2) m^{n-2}\right\}^{1 / 2}(\alpha e)^{m} m^{-m} \\
& =O\left(\left\{(m !)^{-1} n(n+2) \ldots(n+2 m-2) m^{n-1}\right\}^{1 / 2} \alpha^{m}\right) \quad(m \rightarrow \infty),
\end{aligned}
$$

by Stirling's formula. When $m \geq 1$,

$$
(m !)^{-1} n(n+2) \ldots(n+2 m-2)=2^{m}\left(1+\frac{\frac{1}{2} n-1}{m}\right)\left(1+\frac{\frac{1}{2} n-1}{m-1}\right) \ldots\left(1+\frac{\frac{1}{2} n-1}{1}\right)
$$


and

$$
\log \left\{\left(1+\frac{\frac{1}{2} n-1}{m}\right)\left(1+\frac{\frac{1}{2} n-1}{m-1}\right) \ldots\left(1+\frac{\frac{1}{2} n-1}{1}\right)\right\} \leq\left(\frac{1}{2} n-1\right) \sum_{j=1}^{m} j^{-1} \leq\left(\frac{1}{2} n-1\right)(\log m+1) .
$$

Hence

$$
(m !)^{-1} n(n+2) \ldots(n+2 m-2) m^{n-1}=O\left(2^{m} m^{3 n / 2-2}\right) \quad(m \rightarrow \infty),
$$

and the lemma follows.

5. Proofs of Theorems 1 and 2. If $h$ satisfies the hypotheses of Theorem 1 , then, by Lemma 1,

$$
D^{a} h(O) \rightarrow 0 \quad(|a| \rightarrow \infty)
$$

Hence there exists a non-negative integer $q$ such that $D^{a} h(O)=0$ whenever $|a| \geq q$. It follows that the multiple Taylor series of $h$ about $O$ has only finitely many non-zero terms and hence that $h$, being equal in $\mathbf{R}^{n}$ to the sum of this series (see e.g. [4]), is a polynomial.

If $h$ satisfies the hypotheses of Theorem 2, then by Lemma 2,

$$
\left|\nabla_{m} h(O)\right| \rightarrow 0 \quad(m \rightarrow \infty)
$$

Hence there exists a non-negative integer $q$ such that $\left|\nabla_{m} h(O)\right|=0$ when $m \geq q$. This implies that $D^{a} h(O)=0$ when $|a| \geq q$ and hence that $h$ is a polynomial.

Consideration of the functions $h_{1}$ and $h_{2}$, defined in $\mathbf{R}^{n}$ by the equations

$$
h_{1}(X)=e^{x_{1}} \cos x_{2}
$$

and

$$
h_{2}(X)=e^{x_{1} / \sqrt{2}}\left\{\cos \left(x_{2} / \sqrt{2}\right)+\sin \left(x_{2} / \sqrt{2}\right)\right\}
$$

shows that Theorems 1 and 2 fail with $\alpha=1$ and $\alpha=1 / \sqrt{2}$, respectively. The verifications are left to the reader.

\section{REFERENCES}

1. D. H. Armitage, Uniqueness theorems for harmonic functions which vanish at lattice points, J. Approximation Theory (to appear).

2. M. Brelot and G. Choquet, Polynômes harmoniques et polyharmoniques, Deuxième colloque sur les équations aux derivées partielles, Bruxelles (1954), 45-66.

3. A. P. Calderón and A. Zygmund, On higher gradients of harmonic functions, Studia Math. 24 (1964), 211-226. 
4. W. K. Hayman, Power series expansions for harmonic functions, Bull. London Math. Soc. 2 (1970), 152-158.

5. L. L. Helms, Introduction to potential theory (New York, 1969).

6. Ü. Kuran, On norms of higher gradients of harmonic functions, J. London Math. Soc. (2) 3 (1971), 761-766.

7. Ü. Kuran, On Brelot-Choquet axial polynomials, ibid. (2) 4 (1971), 15-26.

8. C. Müller, Spherical harmonics, Lecture Notes in Mathematics, No. 17, (Springer-Verlag, 1966).

9. G. Pólya, Über die kleinsten ganzen Funktionen, deren sämtliche Derivierten im Punkte $z=0$ ganzzahlig sind, Tôhoku Math. J. 19 (1921), 65-68.

10. E. G. Straus, On entire functions with algebraic derivatives at certain algebraic points, Ann. of Math. 52 (1950), 188-198.

The Queen's University,

BELFAST BT7 1NN. 